\title{
UV Direct Laser Interference Patterning of Diffraction Gratings in Poly- Hydroxyethyl-Methacrylate Ophthalmic Polymers
}

\author{
Daniel Sola ${ }^{* 1,2}$, Sabri Alamri ${ }^{3}$ and Andrés F. Lasagni ${ }^{1,3}$ \\ ${ }^{1}$ Institut für Fertigungstechnik, Technische Universität Dresden, 01062 Dresden, Germany \\ ${ }^{2}$ Aragonese Foundation for Research and Development (ARAID), Government of Aragon, 50018 \\ Zaragoza, Spain \\ ${ }^{3}$ Fraunhofer-Institut für Werkstoff- und Strahltechnik IWS, 01277 Dresden, Germany \\ *Corresponding author: daniel.sola@tu-dresden.de,dsola@unizar.es
}

\begin{abstract}
Laser-induced refractive index modification by using micro-structuration of diffractive optical devices in ophthalmic polymers is of great interest in the fields of Optics and Ophthalmology. In this work, poly-hydroxyethyl-methacrylate, PHEMA, polymers used as soft contact lenses were structured with linear periodic patterns by means of Direct Laser Interference Patterning, DLIP. Periodic modulation on the surface of the polymer material was attained under two-beam interference with a Q-switched laser source with emission at $266 \mathrm{~nm}$ and $10 \mathrm{~ns}$ pulse duration. Features of the laser processed areas were studied as a function of both the laser fluence and the interference period. Topography of structured areas was investigated using optical confocal microscopy. Compositional and structural modifications of the processed material were studied by means of micro-Raman spectroscopy, and scanning electron microscopy, SEM, confirming that at low laser fluences structured material remained unaltered. Finally, both diffractive properties and refractive index modification were assessed through diffractive techniques under illumination of a continuous-wave $632.8 \mathrm{~nm} \mathrm{He}-\mathrm{Ne}$ laser resulting in values ranging $7.8 \times 10^{-2}$ and $5.6 \times 10^{-2}$.
\end{abstract}

DOI: $10.2961 /$ jlmn.2020.03.2005

Keywords: direct laser interference patterning, laser materials processing, surface structuring, polymers, diffraction gratings.

\section{Introduction}

Short and ultrashort laser pulses have been widely applied to fabricate two- and three-dimensional permanent structures inside transparent optical materials such as waveguides, photonic crystals, diffraction gratings, beam splitters, etc. [1-7]. During the last decade ultrafast laser inscription, ULI, has been proposed as a new approach to change the power of refractive optical elements for ophthalmic applications. In particular, linear diffraction gratings were inscribed within dye-doped and non-doped ophthalmic polymers, exvivo and in-vivo corneal stroma by using high-repetitionrate ultrashort laser pulses with pulse energy below damage threshold. Refractive index modification achieved ranged $6 \times 10^{-2} \sim 8 \times 10^{-2}$ in polymers [8-11]. Nevertheless, the time required to process areas of similar dimensions to that of the cornea, even at the maximal scanning speed reported to date, $20 \mathrm{~mm} / \mathrm{s}$ [10], is too long and makes this technique inviable to be applied at real scale. This technological limitation may be overcome by using direct laser interference patterning, DLIP. This single-step and non-contact laser processing technique consists in the interference of two or more pulsed laser beams to generate a spatial variation of intensity which is transferred to the substrate to create periodic patterns with controlled dimensions. Structured geometry is managed by the interference angle, the laser wavelength, and the laser intensity. This technique has been used to fabricate periodic arrays in a wide range of metals and polymers and has been demonstrated to be more flexible and cost-effective when compared to traditional micro- and sub-micrometer structuring methods [12-18].

In this work fabrication and characterization of diffractive gratings in poly-hydroxyethyl-methacrylate polymers used as soft contact lenses by using direct laser interference patterning, DLIP, is presented. Diffraction gratings were structured in dry stage by using a pulsed laser source emitting at $266 \mathrm{~nm}$ with pulsewidth in the nanosecond range. The surface topography of the irradiated areas was investigated using optical confocal microscopy, bright field and phase contrast microscopy, and Scanning Electron Microscopy, SEM. Also, structural modification was studied by means of micro-Raman spectroscopy. Finally, periodic patterns were characterized by diffractive techniques to ascertain the diffractive properties of the DLIP periodic patterns.

\section{Experimental}

\subsection{Material}

Ophthalmic poly-hydroxyethyl-methacrylate, PHEMA$\mathrm{UV}$, was used as substrate. This polymer when used for soft contact lenses usually incorporates UV filters which shift the 
absorption edge to $375 \mathrm{~nm}$, as shown in Figure 1. Samples were $1 \mathrm{~mm}$ thick and were processed in dry stage.

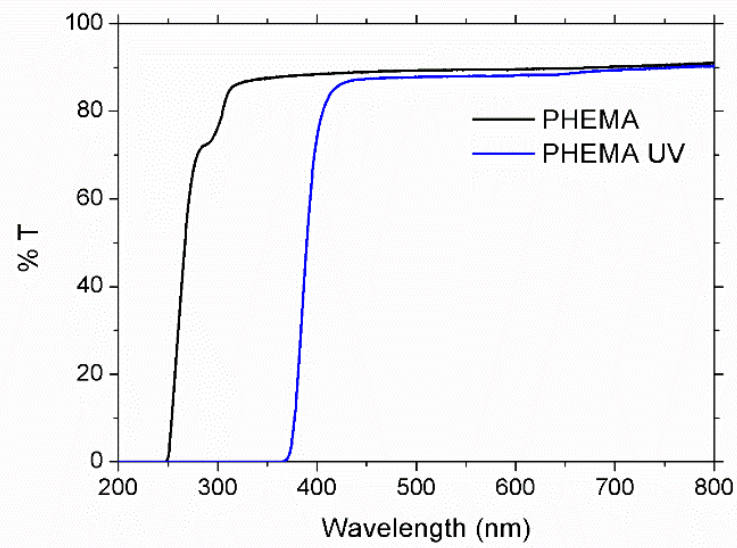

Fig. 1 Optical transmission spectra of PHEMA and PHEMA-UV polymer.

\subsection{Laser structuring}

As the laser source a Q-Switched Nd:YAG laser emitting $10 \mathrm{~ns}$ pulses at a wavelength of $266 \mathrm{~nm}$ with a repetition rate of $10 \mathrm{~Hz}$ was used to fabricate the periodic structures (Quanta Ray, Spectra Physics). Laser processing was carried out under the two-beam configuration, as shown in Figure 2. Laser beam was split into two beams of equal intensity by a beam splitter and the optical path of both beams was adjusted by mirrors and lenses. The interference period, $\Lambda$, was controlled by the angle between the laser beams, $2 \alpha$, and the laser wavelength, $\lambda$, of the laser light according to the following equation [12]:

$$
\Lambda=\frac{\lambda}{2 \sin \alpha}
$$

Interference periods were set at $2.6 \mu \mathrm{m}$ and $4.7 \mu \mathrm{m}$. Features of the laser processed areas were also studied as a function of the laser fluence, delivering fluences on the surface of the samples between $0.5 \mathrm{~J} / \mathrm{cm}^{2}$ and $17 \mathrm{~J} / \mathrm{cm}^{2}$, and number of pulses, between 1 and 5 pulses. To structure large areas, a $0.5 \mathrm{~mm}$ side square mask was used to obtain a square pixel and the sample was translated in $x$ and $y$ directions controlling the distance between adjacent pixels. Since the mask was located at a significant distance from the laser source, the shape of the beam at this position can be considered Gaussian. Then, two $100 \mathrm{~mm}$ optical lenses were used to focus the interference pattern on the surface of the sample, as shown in Figure 2 (double lens system).

\subsection{Characterization techniques}

Superficial topography and profile measurements were carried out using an optical confocal microscope (Sensofar $\mathrm{S}$ Neox). Bright field and phase contrast images were taken using a phase contrast microscope (M-800, Optika). Microstructural analyses were determined by environmental Field Emission Gun Scanning Electron Microscopy, FEG-SEM, (Quanta FEG-250) with Energy Dispersive X-ray Spectroscopy, EDX, detector incorporated. Micro-Raman dispersion measurements were performed using a spectrograph (SR303i-B, Andor) equipped with a thermoelectric-cooled CCD detector (DU920P-BEX2-DD, Andor). As the excitation source, a $532 \mathrm{~nm}$ laser was used, and the scattered light was collected in confocal configuration through a $60 \times(\mathrm{NA}=$ $0.85)$ microscope objective lens. The output power of the laser was kept below $25 \mathrm{~mW}$ in order to avoid significant local heating of the samples. A continuous-wave $3 \mathrm{~mW} \mathrm{He}-\mathrm{Ne}$ laser at $632.8 \mathrm{~nm}$ was used to illuminate the DLIP periodic patterns to characterize the diffractive modes.

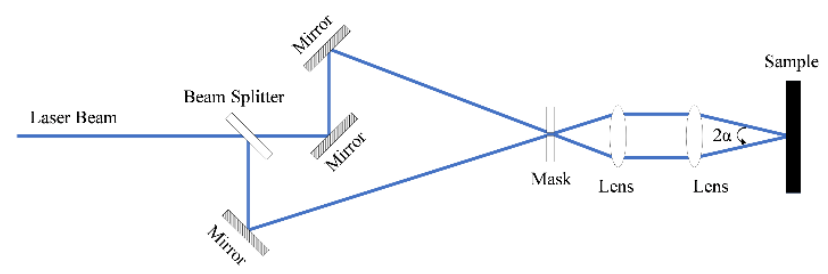

Fig. 2 Schematic representation of the DLIP two-beam interference configuration setup.

\section{Results and discussion}

\subsection{DLIP structuring}

Periodic line-like patterns arrays were produced on the polymer samples using a two-beam laser interference setup, for which the modulation of the intensity profile experienced on the surface of the sample can be described according to the equation [12]:

$$
I_{P}(x, y)=4 I_{L} \cos ^{2}\left(\frac{2 \pi}{\lambda} x \sin \alpha\right)
$$

where, $x$ and $y$ are the coordinate axes in the perpendicular and parallel directions to the linear pattern respectively, $I_{L}$ is the laser fluence of each beam, $2 \alpha$ the interference angle and $\lambda$ the laser wavelength. This distribution of intensity may be transferred onto the material surface by photo-thermal, photo-chemical or photo-physical processes. Considering the high absorption of the polymer samples at the laser wavelength used to carry out the process, $266 \mathrm{~nm}$, and that the pulse duration is in the nanosecond range, $10 \mathrm{~ns}$, both photo-chemical and photo-thermal processes will take part in the structuring process implying direct bond breaking and thermally induced vaporization processes [19].

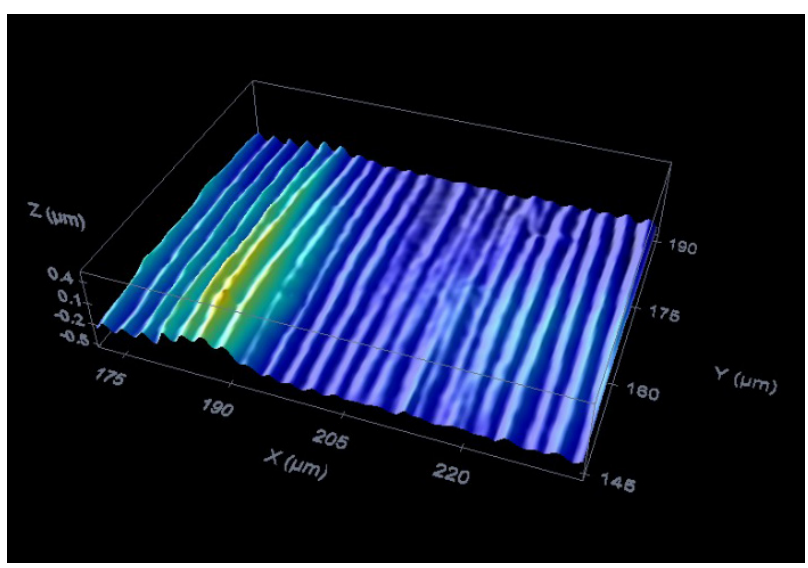

Fig. 3 Confocal image of a sample structured with a period of $2.6 \mu \mathrm{m}$ obtained using one laser pulse and a laser fluence of $7 \mathrm{~J} / \mathrm{cm}^{2}$

The experimental setup was adjusted according to the Equation 1 to structure both types of polymer samples with 
spatial periods of $2.6 \mu \mathrm{m}$ and $4.7 \mu \mathrm{m}$. Single-pulse laser structuring was assessed modifying the laser fluence between $7 \mathrm{~J} / \mathrm{cm}^{2}$ and $17 \mathrm{~J} / \mathrm{cm}^{2}$. In addition, multi-pulse laser structuring was investigated delivering 1,3 and 5 pulses at a fluence of $17 \mathrm{~J} / \mathrm{cm}^{2}$. As an example, Figure 3 shows the surface topography of a sample structured with a period $\Lambda$ of $2.6 \mu \mathrm{m}$ obtained using one laser pulse and a laser fluence of $7 \mathrm{~J} / \mathrm{cm}^{2}$. Profile measurements carried out in the processed areas by confocal microscopy allowed determining the experimental period of the interference pattern, resulting in $2.64 \pm 0.15 \mu \mathrm{m}$ and $4.78 \pm 0.48 \mu \mathrm{m}$, in good agreement with the theoretical calculation provided by Equation 1 .

At low laser fluences, it was observed that laser-matter interaction process resulted in the swelling of the polymer surface, as shown in Figure 3. Swelling is a pre-ablative process commonly observed at low fluences, which consists in the softening of the irradiated surface giving rise to the material expansion. This process has been observed under certain conditions and may be attributed to both a local heating of the polymer sample and the decomposition of the UV absorbers of the polymer sample, which results in molecular nitrogen elimination and eventually to other gaseous byproducts [15, 19-22]. This phenomenon was observed for laser fluences up to $9 \mathrm{~J} / \mathrm{cm}^{2}$ for a spatial period of $2.6 \mu \mathrm{m}$ and up to $17 \mathrm{~J} / \mathrm{cm}^{2}$ for a spatial period of $4.7 \mu \mathrm{m}$. At higher laser fluences, material structuring was produced by ablation mechanisms.

The height of DLIP structures increased with the laser fluence in both single-pulse and multi-pulse modes, as shown in Figure 4. Furthermore, as previously reported, the height of the periodic structure decreased when the spatial period was decreased from $4.7 \mu \mathrm{m}$ to $2.6 \mu \mathrm{m}$ [15]. Nevertheless, in addition to structured areas a heat affected zone, HAZ, appeared in the processed areas for laser fluences higher than $11 \mathrm{~J} / \mathrm{cm}^{2}$ for a spatial period of $2.6 \mu \mathrm{m}$ and 51 $\mathrm{J} / \mathrm{cm}^{2}$ for a spatial period of $4.7 \mu \mathrm{m}$. This was because of the photothermal-mechanical nature of the laser-matter interaction process. As an example, Figure 5 shows a top-view SEM micrograph of a sample processed with a single pulse at a fluence of $11 \mathrm{~J} / \mathrm{cm}^{2}$ and a spatial period of $2.6 \mu \mathrm{m}$ in which the molten material produced during the ablation process and redeposited on the interaction zone is observed.

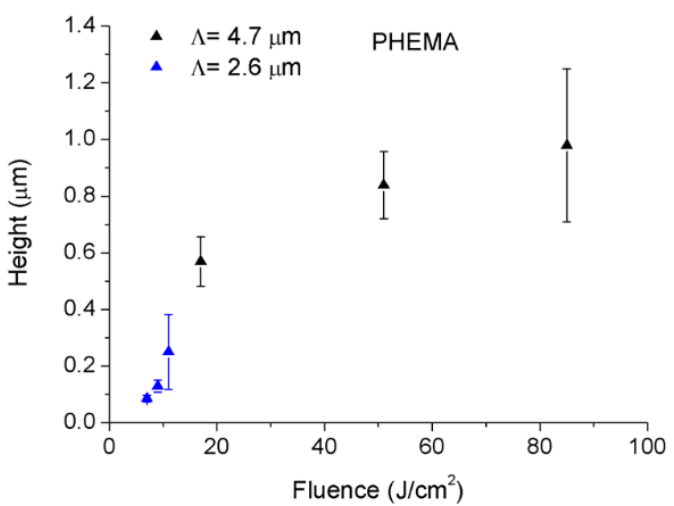

Fig. 4 Height achieved in DLIP-structured PHEMA sample.

\subsection{Microstructural characterization}

Chemical composition and structural analyses of the laser treated polymers were also investigated by $\mu$-Raman spectroscopy. Figure 6 shows Raman spectra in non-processed areas and in the DLIP structured regions at both low and high laser fluences. Raman spectra were made up of sharp peaks and broad bands which can be assigned as follows [23, 24]: $473 \mathrm{~cm}^{-1}$, deformation mode; $604 \mathrm{~cm}^{-1}$, vsCCO; $830 \mathrm{~cm}^{-1}$, vsCOC; $897 \mathrm{~cm}^{-1}$, vsCOC $(\mathrm{H}) ; 968 \mathrm{~cm}^{-1}$, $\rho \mathrm{CH}_{3} ; 1029 \mathrm{~cm}^{-1}, v \mathrm{CC} ; 1089 \mathrm{~cm}^{-1}$, vasOCH${ }_{2} \mathrm{C}, \rho \mathrm{CH} 3$, and $\rho_{\mathrm{CH}_{2}} ; 1204 \mathrm{~cm}^{-1}, \tau \mathrm{CH}_{2}$ and $\omega \mathrm{CH}_{2} ; 1277 \mathrm{~cm}^{-1}, \tau \mathrm{CH}_{2}$ and $\omega \mathrm{CH}_{2} ; 1455 \mathrm{~cm}^{-1}, \delta \mathrm{CH}_{2}$ and $\delta \mathrm{CH}_{3}$; and $1718 \mathrm{~cm}^{-1}, v \mathrm{C}=\mathrm{O}$.

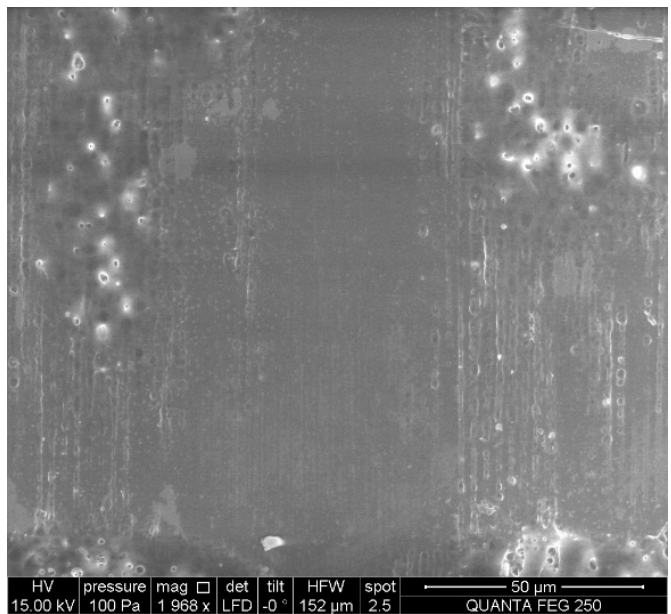

Fig. 5 Top-view SEM micrograph of a sample processed with a single pulse at a fluence of $11 \mathrm{~J} / \mathrm{cm}^{2}$ and a spatial period of $2.6 \mu \mathrm{m}$.

Raman spectra of DLIP structured areas processed at high fluence showed significant variations when compared to that corresponding to the non-processed samples. In particular, at high laser fluence there was a strong decrease of the peak intensity in the components at $473 \mathrm{~cm}^{-1}, 604 \mathrm{~cm}^{-1}$, $830 \mathrm{~cm}^{-1}, 897 \mathrm{~cm}^{-1}, 1089 \mathrm{~cm}^{-1}$ and $1718 \mathrm{~cm}^{-1}$, whereas the components at $734 \mathrm{~cm}^{-1}, 1204 \mathrm{~cm}^{-1}, 1230 \mathrm{~cm}^{-1}$ disappeared. Conversely, at low fluence the laser structuring was not accompanied with important changes in the Raman spectra and hence the polymer structure remained almost unaltered after laser irradiation.

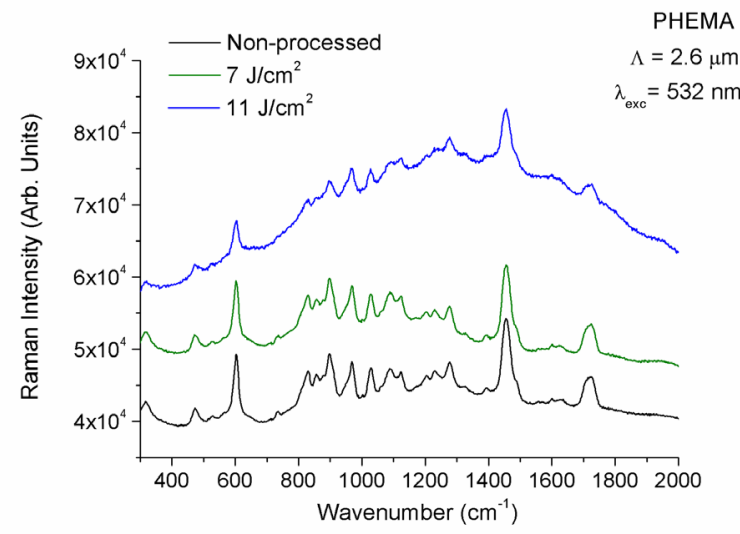

Fig. 6 Micro-Raman spectra of PHEMA samples.

Mechanisms responsible for refractive index changes may be attributed to photodecomposition, local heat accumulation, dehydration, additional cross-linking and tensile stress [25-27]. The strong fluorescence background increase 
observed in samples processed at high fluence can be assigned to thermal decomposition of organic molecules [25]. On the other hand, when laser processed areas do not present significant changes in the structure of the polymer matrix, such as samples processed at low laser fluence, the refractive index mechanism can be attributed to additional cross-linking and local densification [25].

\subsection{Optical characterization}

Refractive index changes achieved in the DLIP periodic patterns structured were characterized under illumination of a continuous-wave He-Ne laser at $632.8 \mathrm{~nm}$. DLIP periodic patterns showed diffraction patterns at both spatial periods, $4.7 \mu \mathrm{m}$ and $2.6 \mu \mathrm{m}$, with diffraction angles in good agreement with the diffraction equation $m \lambda=\Lambda \sin \theta$, where $m$ is the diffraction order, $\lambda$ the laser wavelength and $\Lambda$ the grating period. As an example, Figure 7 shows far-field diffraction pattern obtained from single-pulse mode, $17 \mathrm{~J} / \mathrm{cm}^{2}$ laser fluence, and $\Lambda=4.7 \mu \mathrm{m}$ structuring period.

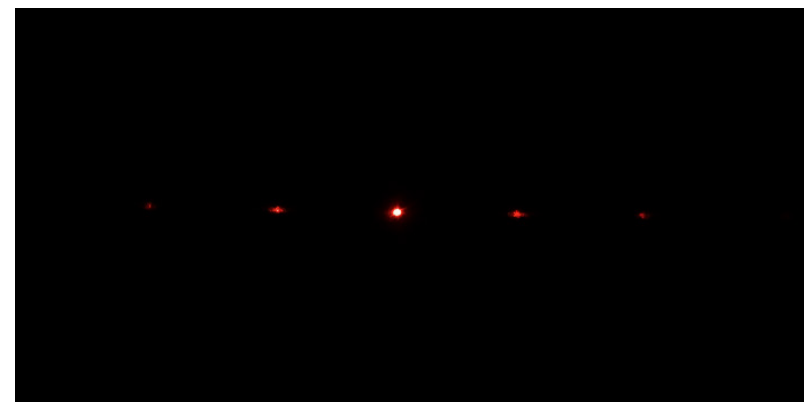

Fig. 7 Diffraction images of DLIP periodic patterns obtained in single-pulse mode at a fluence of 17 $\mathrm{J} / \mathrm{cm}^{2}$ and a structuring period $\Lambda=4.7 \mu \mathrm{m}$.

Next, zero- and first-order intensity of each diffracted order were measured by using a power-meter providing a firstorder efficiency, $\mathrm{I}_{1} / \mathrm{I}_{0}$, of 0.0013 and 0.0307 for samples processed at $14.0 \mathrm{~J} / \mathrm{cm}^{2}$ and $17 \mathrm{~J} / \mathrm{cm}^{2}$, respectively. At this laser fluences total efficiency of the diffraction gratings were found to be higher than $98 \%$ so that refractive index change could be determined considering the diffraction grating as a phase grating. In addition, refractive index change was assumed to be both uniform and top-hat shaped within the irradiated region. With these assumptions the intensity of the $0^{\text {th }}$ and $1^{\text {st }}$ order diffracted light can be expressed as [8]:

$$
I_{0}=\left(\frac{1}{\lambda z}\right)^{2}\left[\left(e^{i 2 \pi \frac{(n+\Delta n) b}{\lambda}}-e^{i 2 \pi \frac{n b}{\lambda}}\right) \frac{a}{\Lambda}+e^{i 2 \pi \frac{n b}{\lambda}}\right]^{2}
$$

and

$$
I_{1}=\left(\frac{1}{\lambda z}\right)^{2}\left[\left(e^{i 2 \pi \frac{(n+\Delta n) b}{\lambda}}-e^{i 2 \pi \frac{n b}{\lambda}}\right) \frac{a}{\Lambda} \sin c\left(\frac{a}{\Lambda}\right)\right]^{2}
$$

where $\lambda$ is the laser wavelength, $n$ the refractive index of the material, $b$ the thickness of the grating line, $a$ the width of the grating line and $\Lambda$ the grating period. First-order efficiency provided by the ratio of Equations 4 and 3 was plotted versus the refractive index change, $\Delta n$. For the first-order efficiencies obtained in the samples the corresponding refractive index changes were found to be $7.8 \times 10^{-2}$ and $5.6 \times 10^{-2}$ for laser fluences at $14 \mathrm{~J} / \mathrm{cm}^{2}$ and $17 \mathrm{~J} / \mathrm{cm}^{2}$, respectively. These values were slightly higher to those previously reported by using the ULI technique in silicone and non-silicone based hydrogel polymers $6 \times 10^{-2}[8]$, and in the same order of magnitude in dye-doped silicone hydrogels $8 \times 10^{-2}$ [9]. It is worth highlighting that these gratings were manufactured at $0.4 \mu \mathrm{m} / \mathrm{s}$ and $600 \mu \mathrm{m} / \mathrm{s}$ for the non-doped and doped polymers respectively so that the fabrication yields for a grating with a spatial period of $4.7 \mu \mathrm{m}$ would be $1.88 \times 10^{-6} \mathrm{~mm}^{2} / \mathrm{s}$ and $2.82 \times 10^{-3} \mathrm{~mm}^{2} / \mathrm{s}$ respectively, whereas the fabrication yield for the same grating manufactured by DLIP in the experimental conditions studied in this work ranges $0.23 \mathrm{~mm}^{2} / \mathrm{s}$ and $2.2 \mathrm{~mm}^{2} / \mathrm{s}$, more than two orders of magnitude faster.

\section{Conclusions}

Direct laser interference patterning was successfully applied to structure PHEMA polymers used as soft contact lenses. A Q-switched laser delivering $10 \mathrm{~ns}$ laser pulses at $266 \mathrm{~nm}$ and the two-beam configuration setup was used to fabricate periodic line-like patterns with spatial periods of $2.6 \mu \mathrm{m}$ and $4.7 \mu \mathrm{m}$. At low laser fluences it was observed that laser-matter interaction process resulted in the swelling of the polymer surface. As the laser fluence increased the height of the DLIP structure also increased in both singlepulse and multi-pulse processing modes. Furthermore, the height of the periodic structure decreased when decreasing the spatial period. However, high laser fluences achieved at both single and multi-pulse modes induced a heat affected zone in which the material removed from the surface during the laser interaction process was redeposited on the interaction zone. Micro-Raman analyses carried out in the processed areas showed that at low laser fluence the material remained almost unaltered. On the contrary, at high laser fluence the material underwent degradation with significant changes in the chemical structure showing a strong fluorescence background attributed to thermal decomposition of organic molecules. DLIP structured areas showed diffraction patterns at both spatial periods, $4.7 \mu \mathrm{m}$ and $2.6 \mu \mathrm{m}$. $1^{\text {st }}$ to $0^{\text {th }}$ order diffracted light efficiency was used to assess the refractive index modification, resulting in $7.8 \times 10^{-2}$ and $5.6 \times 10^{-2}$ for $14 \mathrm{~J} / \mathrm{cm}^{2}$ and $17 \mathrm{~J} / \mathrm{cm}^{2}$ laser fluences, respectively. Refractive index mechanism for these low scattering loss DLIP gratings can be attributed to additional cross-linking and local densification. These values of refractive index change were similar to those reported by using the ULI technique but with an improvement of the processing yield of more than two orders of magnitude.

\section{Acknowledgments}

This research was funded by European Union's Horizon 2020 research and innovation programme under the Marie Skłodowska-Curie IF No 795630. Fundación Séneca grant No $20647 / \mathrm{JLI} / 18$ is also acknowledged. The work of S. Alamri has been supported by the European Union's Horizon 2020 research and innovation programme under the Marie Skłodowska-Curie grant agreement No 675063. The work of A.F. Lasagni is also supported by the German Research Foundation (DFG) under Excellence Initiative program by the German federal and state governments to promote top-level research at German universities. 


\section{References}

[1] H. Misawa and S. Juodkazis: "3D laser microfabrication" (Wiley, 2006).

[2] K.M. Davis, K. Miura, N. Sugimoto, and K. Hirao: Opt. Lett., 21, (1996) 1729.

[3] S. Nolte, M. Will, J. Burghoff, and A. Tuennermann: Appl. Phys. A, 77, (2003) 109.

[4] D. Sola, A. Escartín, R. Cases, and J.I. Peña: Opt. Mater., 33, (2011) 728.

[5] D. Sola, J. Martinez de Mendibil, J.R. Vazquez de Aldana, G. Lifante, R. Balda, A.H. de Aza, P. Pena, and J. Fernandez: Appl. Surf. Sci., 278, (2013) 289.

[6] F. Chen and J.R. Vazquez de Aldana: Laser Photon. Rev., 8, (2014) 251.

[7] J. Martinez de Mendivil, D. Sola, J.R. Vazquez de Aldana, G. Lifante, A.H. de Aza, P. Pena, and J.I. Peña: J. Appl. Phys., 117, (2015) 043104.

[8] L. Ding, R. Blackwell, J.F. Künzler, and W.H. Knox: Opt. Express, 14, (2006) 11901.

[9] L. Ding, D. Jani, J. Linhardt, J.F. Künzler, S. Pawar, G. Labenski, T. Smith, and W.H. Knox: Opt. Express, 16, (2008) 21914.

[10]L. Ding, W.H. Knox, J. Bühren, L.J. Nagy, and K.R. Huxlin: Investig. Ophthalmol. Vis. Sci., 49, (2008) 5332.

[11]K.T. Wozniak, S.M. Gearhart, D.E. Savage, J.D. Ellis, W.H. Knox, and K.R. Huxlin: J. Biomed. Opt., 22, (2017) 055007.

[12]L. Müller-Meskamp, Y.H. Kim, T. Roch, S. Hofmann, R. Scholz, S. Eckardt, K. Leo, and A.F. Lasagni: Adv. Mater., 24, (2012) 906.

[13]M. Bieda, M. Siebold, and A.F. Lasagni: Appl. Surf. Sci., 387, (2016) 175.
[14] V. Lang, T. Roch, and A.F. Lasagni: Adv. Eng. Mater., 18, (2016) 1342.

[15] S. Alamri and A.F. Lasagni: Opt. Express, 25, (2017) 9603.

[16] S. Alamri, A.I. Aguilar-Morales, and A.F. Lasagni: Eur. Polym. J., 99, (2018) 27.

[17]D. Sola, C. Lavieja, A. Orera, and M.J. Clemente: Opt. Lasers Engin., 106, (2018) 139.

[18]D. Sola, S. Alamri, A.F. Lasagni, and P. Artal: Appl. Surf. Sci., 476, (2019) 128.

[19]R. Srinivasan and B. Braren: Chem. Rev., 89, (1989) 1303.

[20] R. Srinivasan and B. Braren, K.G. Casey: J. Appl. Phys., 68, (1990) 1842.

[21]F. Beinhorn, J. Ihlemann, K. Luther, and J. Troe: Appl. Phys. A, 68, (1999) 709.

[22]T. Roch, F. Klein, K. Guenther, A. Roch, T. Mühl, and A. Lasagni: Mater. Res. Expr., 1, (2014) 035042.

[23]P. Taddei, F. Balducci, R. Simoni, and P. Monti: J. Mol. Struct., 744-747, (2005) 507.

[24] A. Bertoluzza, P. Monti, J.V. Garcia-Ramos, R. Simoni, R. Caramazza, and A. Calzavara: J. Mol. Struct., 143, (1986) 469.

[25] L. Ding, L.G. Cancado, L. Novotny, W.H. Knox, N. Anderson, D. Jani, J. Linhardt, R.I. Blackwell, and J.F. Künzler: J. Opt. Soc. Am. B, 26, (2009) 595.

[26]P.J. Scully, D. Jones, and D.A. Jaroszynski: J. Opt. APure Appl. Opt., 5, (2003) S92.

[27]H. Mochizuki, W. Watanabe, Y. Ozeki, K. Itoh, K. Matsuda, and S. Hirono: Thin Solid Films, 518, (2009) 714.

(Received: June 22, 2020, Accepted: October 8, 2020) 